\title{
Short ribosomal prophylaxis in the prevention of clinical recurrences of chronic otitis media in children
}

\author{
Mora Renzo*, Ralli Giovanni, Passali Francesco Maria, Crippa Barbara, \\ Ottoboni Stefano, Mora Francesco, Barbieri Marco
}

\author{
ENT Department, University of Genoa, Via dei Mille 11/9, Genoa 16147, Italy
}

Received 8 June 2003; received in revised form 5 September 2003; accepted 8 September 2003

\section{KEYWORDS \\ Children; \\ Otitis media; \\ Ribosomal therapy}

\begin{abstract}
Summary The aim of this study was to compare the efficacy and the safety of a short oral ribosomal immunotherapy (Immucytal) in the prevention of chronic otitis media in children. Seventy-two patients were enrolled in this study, 41 males and 31 females, aged between 6 and 14 years, with an history of recurrent otitis media. Patients were randomised to receive Immucytal (group A) or placebo (group B) according to the following protocol: one tablet daily in the morning 8 days per month for three consecutive months. Immucytal and placebo were identical in shape and size, in order to maintain double-blind conditions. The efficacy parameters were (evaluated before, at the end and 6 months after the beginning of the therapy): clinical score; changes in immunological parameters; patient's parents assessment of symptoms on a scale from 0 (much worse) to 4 (much improved) and hearing tests. Patients of group A, had an improvement of clinical items measured, serum concentrations of immunoglobulins, subjective patient's parents assessment of symptoms and hearing tests. For all evaluations, a significant difference between treatment groups was found. Using this dosage and posology (shorter than others) the beneficial effects of Immucytal were maintained until the end of the 6-month study period.
\end{abstract}

(c) 2003 Elsevier Ireland Ltd. All rights reserved.

\section{Introduction}

Otitis media with effusion (OME) is an inflammatory condition characterized by the presence of secretion in the middle ear behind an intact tympanic membrane.

Clinically otitis media presents in several forms that are characterized by both the duration of the disease and the type of exudate. The usual symptomatic presentation is known as acute otitis media

\footnotetext{
${ }^{*}$ Corresponding author. Tel.: +39-010-3537631; fax: + 39-010-3537684

E-mail address: renzomora@libero.it (M. Renzo).
}

(AOM) and is characterized often by fever, irritability, lethargy, vomiting, diarrhea and hearing loss in some children. However, it is important to realize that in up to half of the children who have tympanic membrane signs of otitis media, they may not have symptoms which demonstrate the insidious nature of the disease. If it persists for more than 3 months, it is considered to be chronic [1].

OME is considered the most common disease of the childhood and there is no sex preponderance. About $80 \%$ of children are affected by OME at least once in their childhood and the highest incidence of this disease occurs between 1 and 5 years of age [2]. 
The pathogenesis of AOM involves a complex interplay between viruses, bacteria and the host's inflammatory response [3].

Several studies ranging from animal experiments to extensive clinical trials supports a crucial role for respiratory viruses in the etiology and pathogenesis of otitis media [4,5].

Viral infection of the upper respiratory mucosa begins the whole cascade of events that finally leads to the development of acute otitis media as a complication. Recent data indicate that at least some types of viruses (respiratory syncytial virus, parainfluenza viruses, influenzaviruses, enteroviruses, adenoviruses) actively invade the middle ear and may also interfere with the outcome of otitis media [5-7].

Although viruses are usually the primary pathogens involved, superinfections with bacteria are frequent. The most common bacteria involved in such infections are haemophilus influenzae, streptococcus pneumoniae, haemophilus parainfluenzae, streptococcus pyogenes, staphylococcus aureus and moraxella (branhamella) catarrhalis [5-7].

The current management of otitis media is based on curative and preventive measures (mainly antibacterial and symptomatic therapies). Antibacterial therapy is still the classical treatment approach in patients with otitis media. With concerns growing about increased microbial antibiotic resistance, attention has been focused on the possibility of vaccination against some of the pathogens responsible [2].

In contrast to antibacterials, ribosomal immunotherapy is designed to enhance both specific and non-specific immunity against the most common pathogens involved in respiratory tract infections. In the last years, several studies have demonstrated the immunostimulant activity of various bacterial extracts, which seems to target not only the bacteria from which such extracts are made but also (trough non-specific mechanism) other pathogens responsible for the most respiratory tract infections $[3,8]$.

The aim of this study was to compare the efficacy and the safety of an oral ribosomal immunotherapy (Immucytal), shorter than others ribosomal immunotherapy, in the prevention of otitis media in children.

\section{Materials and methods}

The study was performed in Italy, during the period from June 2002 to April 2003.

We had considered as exclusion criteria: anatomic anomalies, others contemporary acute infections,
Table 1 Demographic data and infectious status of the patients

\begin{tabular}{ll}
\hline Patient characteristics & $\begin{array}{l}\text { Number of } \\
\text { patients }\end{array}$ \\
\hline Patients enrolled & 72 \\
Males & 41 \\
Females & 31 \\
Mean age (years) & 7.2 \\
Breast feeding & 8 \\
Attending to kindergarten & 49 \\
Incidents of upper respiratory & 45 \\
$\quad$ airways infections (before & \\
$\quad$ the treatment) & \\
Incidents of middle ear & 33 \\
$\quad$ infections (before the & \\
$\quad$ treatment) & \\
Low socio-economic conditions & 28 \\
Passive smoking & 19 \\
\hline
\end{tabular}

congenital immunodeficiences; ear, nose or throat conditions secondary to gastroesophageal reflux or tooth infection; treatments with immunostimulant or immunosoppressive agents (levamisole, bacterial vaccines or corticosteroids) in the past 6 months, diagnosis of bronchopulmonary disease (pneumonia or bronchiectasis) and allergy to the drug (Immucytal).

In this study were enrolled 72 patients, 41 males and 31 females, of age ranging between 6 and 14 years of age. Patients were included if they had $a>2$ years history of recurrent or chronic respiratory infections (at least five episodes of acute respiratory infections in the last year) and/or had experienced otitis media for more than 3 months (Table 1).

An informed consent was signed at the beginning of the therapy by the parents of the patients.

The patients were divided randomly into two numerically equals groups (groups $A$ and $B$ ) to receive Immucytal (group A) or placebo (group B) according to the following protocol: one tablet daily in the morning 8 days per month for three consecutive months. Immucytal and placebo were identical in shape and size, in order to maintain double-blind conditions.

Immucytal contains both proteoglycans from klebsiella pneumoniae and ribosomes from four different bacterial strains (klebsiella pneumoniae, streptococcus pneumoniae, haemophilus influenzae, streptococcus pyogenes A).

Before, at the end and 6 months after the beginning of the therapy all patients underwent the following instrumental examinations: liminal tonal audiometry; tympanometry; otoacoustic emission with linear click emission; otoacoustic products of distortion; plasma levels of immunoglobulins class 
G, A, M, E (IgG, IgA, IgM, IgE); plasma levels of lymphocyte subpopulations (CD4+, CD8+) and subjective assessment of symptoms on a scale from 0 (much worse) to 4 (much improved) by patient's parents.

Other criterion was the outcome as measured by a clinical score based on the overall assessment of the frequency, duration, severity and social impact of otitis. The following items were scored at each control: number $(1,2$ or $>2)$ and duration $(<3 / 3-6 />6$ days) of episodes of otitis; fever (yes/no); medical consultations (yes/no); disease frequency (once/twice/ > twice per month); ancillary therapy required (none/symptomatic only/antibacterials) and absence from school (none/1-3/>4 days) [9].

\section{Results}

Analysing the results of this study it is important to consider that patients were allowed to receive concomitant medications (including antibacterials) to treat the acute episodes, which may have par- tially contributed to the overall positive outcome observed here.

At the end of the protocol, all patients treated with Immucytal (group A) presented a subjective abatement of symptoms. The mean value of scores on the subjective symptom scale fell from 3.9 to 1.7 (in group $B$, the mean value fell from 3.8 to 2.4 ) (Table 2).

Compared with placebo, Immucytal recipients experienced a significantly improvement of the clinical items measured. The reduction in the incidence of infectious episodes became significant versus placebo from the second visit (at the end of the therapy) for ENT infections ( 0.50 versus 0.67 ; $P>0.02$ ), and from the last control for lower respiratory tract infections $(0.03$ versus $0.17 ; P>0.02)$. At the end point, a significantly improved outcome versus placebo was also observed on the incidence of fever $(P>0.02)$, frequency $(P>0.05)$ and duration $(P>0.05)$ of infectious episodes, ancillary therapies $(P>0.01)$ and school absence $(P>0.05)$. No significant differences between Immucytal and placebo recipients were found for medical

Table 2 Subjective assessment of symptoms, number and duration of episodes of otitis, fever, medical consultations, disease frequency, ancillary therapy required and absence from school before, at the end and 6 months after the beginning of the therapy

\begin{tabular}{|c|c|c|c|c|}
\hline Group & Patients & Baseline & Third month & Endpoint \\
\hline \multicolumn{5}{|c|}{ Subjective assessment of symptoms } \\
\hline Immucytal & 36 & 3.9 & 2.3 & 1.7 \\
\hline Placebo & 36 & 3.8 & 2.9 & 2.4 \\
\hline \multicolumn{5}{|c|}{ Number $(1,2$ or $>2)$ of episodes of otitis } \\
\hline Immucytal & 36 & $>2$ & 2 & 1 \\
\hline Placebo & 36 & $>2$ & $>2$ & 2 \\
\hline \multicolumn{5}{|c|}{ Duration $(<3 / 3-6 />6$ days) of episodes of otitis } \\
\hline Immucytal & 36 & $>6$ & $3-6$ & $<3$ \\
\hline Placebo & 36 & $>6$ & $>6$ & $3-6$ \\
\hline \multicolumn{5}{|l|}{ Fever (yes/no) } \\
\hline Immucytal & 36 & Yes & No & No \\
\hline Placebo & 36 & Yes & Yes & Yes \\
\hline \multicolumn{5}{|c|}{ Medical consultations (yes/no) } \\
\hline Immucytal & 36 & Yes & Yes & No \\
\hline Placebo & 36 & Yes & Yes & No \\
\hline \multicolumn{5}{|c|}{ Disease frequency (once/twice/>twice per month) } \\
\hline Immucytal & 36 & > Twice & Twice & Once \\
\hline Placebo & 36 & > Twice & $>$ Twice & Twice \\
\hline \multicolumn{5}{|c|}{ Ancillary therapy required (none/symptomatic only/antibacterials) } \\
\hline Immucytal & 36 & Antibacterials & Symptomatic & None \\
\hline Placebo & 36 & Antibacterials & Antibacterials & Symptomatic \\
\hline \multicolumn{5}{|c|}{ Absence from school (none/1-3/>4 days) } \\
\hline Immucytal & 36 & $>4$ & $1-3$ & None \\
\hline Placebo & 36 & $>4$ & $1-3$ & $1-3$ \\
\hline
\end{tabular}


Table 3 Hearing test improvement $(\mathrm{dB})$ at the end and 6 months after the beginning of the therapy in the two groups

\begin{tabular}{|c|c|c|c|c|}
\hline \multirow[t]{2}{*}{ Frequency $(\mathrm{Hz})$} & \multicolumn{4}{|c|}{ Hearing improvement (mean \pm S.E.M.) } \\
\hline & Group & Patients & Third month & Endpoint \\
\hline 125 & $\begin{array}{l}\text { Immucytal } \\
\text { Placebo }\end{array}$ & $\begin{array}{l}36 \\
36\end{array}$ & $\begin{array}{l}29.8 \pm 3.86 \\
15.6 \pm 4.25\end{array}$ & $\begin{array}{l}28.7 \pm 4.01 \\
12.4 \pm 3.87\end{array}$ \\
\hline 250 & $\begin{array}{l}\text { Immucytal } \\
\text { Placebo }\end{array}$ & $\begin{array}{l}36 \\
36\end{array}$ & $\begin{array}{l}28.9 \pm 3.74 \\
14.8 \pm 2.98\end{array}$ & $\begin{array}{l}29.3 \pm 4.23 \\
11.2 \pm 3.77\end{array}$ \\
\hline 500 & $\begin{array}{l}\text { Immucytal } \\
\text { Placebo }\end{array}$ & $\begin{array}{l}36 \\
36\end{array}$ & $\begin{array}{l}21.1 \pm 2.89 \\
12.7 \pm 4.88\end{array}$ & $\begin{array}{l}19.8 \pm 3.27 \\
12.9 \pm 4.23\end{array}$ \\
\hline 1000 & $\begin{array}{l}\text { Immucytal } \\
\text { Placebo }\end{array}$ & $\begin{array}{l}36 \\
36\end{array}$ & $\begin{array}{l}10.2 \pm 4.86 \\
10.1 \pm 3.56\end{array}$ & $\begin{array}{l}10.3 \pm 4.52 \\
10.4 \pm 3.66\end{array}$ \\
\hline
\end{tabular}

S.E.M.: standard error of the mean.

consultations, which decreased to a similar extent in both groups (Table 2).

At the end point, from an objective point of view, looking at the liminal tonal audiometry test and considering the low frequencies $(125-1000 \mathrm{~Hz})$ : in the Immucytal group 26 patients (72\%) had a complete improvement of their auditory function (21 patients had a type A tympanogram, while 5 patients had a type $C$ tympanogram), 8 patients (22\%) more than $90 \%$ (5 patients had a type A tympanogram and 3 patients a type $C$ tympanogram) and only two patients did not get good results from therapy (these two patients were not presenting specific characteristic to be assembled in a subgroup and they presented a tympanogram types $B$ and $C$ ); in the placebo group 9 patient $(25 \%)$ did not get improvement (six patients had a type B tympanogram and three patients a type $C$ tympanogram), 15 patients (41\%) had an improvement of their auditory function less than $50 \%$ (three patients had a type A tympanogram, nine patients a type $B$ tympanogram and three patients had a type C tympanogram) and 12 patients (33\%) more than $50 \%$ (five patients had a type A tympanogram, three patients had a type $B$ tympanogram and four patients had a type $C$ tympanogram) (Tables 3 and 4).

In the same patients with an improvement of the auditory function, the evoked otoacoustic emissions revealed an improvement from "fail" to "pass" and otoacoustic distortion products, which were previously absent, were evoked at frequencies of the tonal field normally examined.

Serum concentrations of immunoglobulins were significantly increased in Immucytal versus placebo recipients when measured at 3 months (IgG and IgA; $P>0.05$ ) and at the end of the study (IgG and IgA; $P>0.01)$. CD4+ and CD8+ populations were also significantly increased at both endpoints in Immucytal group $(P>0.05$ versus placebo). There was a moderate increase of IgM titers in the active treatment group, which did not reach statistical significance versus placebo (Table 5 ).

No patient experienced side effects from this treatment.

Comparison of groups was made by the unpaired $t$-test, and correlations were analyzed by regression analysis: probability values at less than 0.05 were regarded as significant. Comparisons of groups also were made for repeated measures by analysis of variance.

\section{Discussion}

The current management of otitis is based on antibacterials and symptomatic therapies: these

Table 4 Tympanometry before, at the end and 6 months after the beginning of the therapy in the two groups

\begin{tabular}{|c|c|c|c|c|}
\hline Group & Patients & Baseline & $\begin{array}{l}\text { Third } \\
\text { month }\end{array}$ & Endpoint \\
\hline \multicolumn{5}{|c|}{ Type A tympanogram } \\
\hline Immucytal & 36 & 2 & 24 & 26 \\
\hline Placebo & 36 & 3 & 9 & 8 \\
\hline \multicolumn{5}{|c|}{ Type B tympanogram } \\
\hline Immucytal & 36 & 28 & 7 & 1 \\
\hline Placebo & 36 & 27 & 14 & 18 \\
\hline \multicolumn{5}{|c|}{ Type C tympanogram } \\
\hline Immucytal & 36 & 6 & 5 & 9 \\
\hline Placebo & 36 & 6 & 13 & 10 \\
\hline
\end{tabular}


Table 5 Serum immunoglobulins ( $\mathrm{mg} / \mathrm{dl}$ ) levels and CD4+ and CD8+ counts during treatment with Immucytal and placebo (respectively showing means plus or minus the standard error of the mean)

\begin{tabular}{|c|c|c|c|c|}
\hline Group & Patients & Baseline & Third month & Endpoint \\
\hline \multicolumn{5}{|l|}{$\lg G$} \\
\hline Immucytal & 36 & $1027.05 \pm 198.8$ & $1989.55 \pm 189.6$ & $1822.32 \pm 206.3$ \\
\hline Placebo & 36 & $1165.42 \pm 225.9$ & $\begin{array}{l}1198.26 \pm 216.0 \\
P<0.05\end{array}$ & $\begin{array}{l}1123.07 \pm 228.0 \\
P<0.05\end{array}$ \\
\hline \multicolumn{5}{|l|}{$\lg A$} \\
\hline Immucytal & 36 & $218.13 \pm 107.3$ & $425.64 \pm 111.3$ & $433.99 \pm 113.5$ \\
\hline Placebo & 36 & $210.46 \pm 101.2$ & $\begin{array}{l}224.41 \pm 114.1 \\
P<0.05\end{array}$ & $\begin{array}{l}226.44 \pm 112.3 \\
P<0.05\end{array}$ \\
\hline \multicolumn{5}{|l|}{$\lg M$} \\
\hline Immucytal & 36 & $146.97 \pm 59.31$ & $155.44 \pm 63.03$ & $216.54 \pm 59.06$ \\
\hline Placebo & 36 & $139.68 \pm 66.42$ & $162.69 \pm 48.83$ & $158.89 \pm 50.45$ \\
\hline \multicolumn{5}{|l|}{ CD4+ } \\
\hline Immucytal & 36 & $46.19 \pm 9.01$ & $53.30 \pm 6.93$ & $58.80 \pm 11.73$ \\
\hline Placebo & 36 & $42.63 \pm 9.19$ & $\begin{array}{l}43.80 \pm 5.82 \\
P<0.05\end{array}$ & $\begin{array}{l}46.23 \pm 12.77 \\
P<0.05\end{array}$ \\
\hline \multicolumn{5}{|l|}{$\mathrm{CD} 8+$} \\
\hline Immucytal & 36 & $28.80 \pm 7.29$ & $34.05 \pm 8.61$ & $35.36 \pm 9.76$ \\
\hline Placebo & 36 & $29.82 \pm 6.54$ & $\begin{array}{l}30.75 \pm 4.66 \\
P<0.05\end{array}$ & $\begin{array}{l}31.77 \pm 8.67 \\
P<0.05\end{array}$ \\
\hline
\end{tabular}

S.E.M.: standard error of the mean.

treatments do not prevent the recurrence of infections.

With the emergence of resistant strains and the change over time of the relative distribution of bacteriologic agents known to cause OME, the search of new therapeutic approaches was initiated by several authors.

In this context, preventive strategies, such as ribosomal immunotherapy, may represent a valid alternative approach [3].

Most pathogens gain access to their host through mucosal surfaces. It is therefore desirable to develop vaccination strategies that lead to mucosal immune responses [10].

Ribosomal immunotherapy has been successfully used since the 1960 s to boost the immune system and provide protection against microbial infections. Mucosal immunostimulating may be used both to prevent mucosal infections through the activation of antimicrobial immunity and to treat systemic inflammatory diseases $[11,12]$.

The use of immunostimulating drugs is one way to intervene in the immune system. Many of these agents are of bacterial origin and most are able to stimulate the non-specific immune response by acting on polymorphonuclear cells and macrophages [13].
Ribosomal immunotherapy (Immucytal) contains both proteoglycans from klebsiella pneumoniae and ribosomes from four different bacterial strains (klebsiella pneumoniae, streptococcus pneumoniae, haemophilus influenzae, streptococcus pyogenes A). Because of the presence of proteoglycans and ribosomes from four frequently encountered bacterial strains, "Immucytal" has specific immunostimulant properties. It can stimulate both the non-specific immune response and the specific antibody production $[3,13]$.

This agent (Immucytal) can stimulate the activity of macrophages, polymorphonuclear cells and natural killer cells in leukocytes isolated from human peripheral blood, and to modulate polymorphonuclear leukocyte functions when administered alone or in associations with antibacterials $[14,15]$.

The beneficial effects of Immucytal were apparent, for most parameters, as early as at 3 months after treatment initiation, and were maintained until the end of the 6-month study period. These data confirm that oral immunisation generates rapid and lasting immune response, building increased numbers of memory cells that are readily available to respond to further challenges by either more ribosomal preparations or potential pathogens $[11,16]$. 
The ventilatory function of the eustachian tube (ET) continues to be a main focus of research: in children, tubal constriction is commonly associated with mucopurulent nasal discharge and the inflammation of the pharyngeal orifice of the ET [17].

Other studies highlight the dependence of the clearance function on the active opening function and the effects of viral upper respiratory tract infection on ET function and middle ear pressure [18].

Liminal tonal audiometry, tympanometry and blood tests confirm the induction of specific and non-specific immune responses of the mucosaassociated lymphoid tissue and the consequent and normalization of ET function and middle ear physiology [19].

Treatment of acute otitis media is the leading cause of antibacterial use in children in most developed countries, despite the fact that antibacterials have several well-known drawbacks, especially when used to treat recurrent infections. Amongst other limitations, these drugs are not effective against viral infections, do not enhance the host's defence mechanisms against subsequent infections and, with prolonged use, may induce bacterial resistance [20].

AOM has become increasingly difficult to treat in the 1990s, the decade of drug-resistant pneumococcus. Throughout the world, drug-resistant strains of this pathogen are being recovered from 20 to $50 \%$ of cases of initial untreated AOM and from 45 to $90 \%$ of refractory AOM $[20,21]$.

Rates of streptococcus pneumoniae strains resistant to many classes of antibacterial agents have risen dramatically in many countries over the past 20 years $[20,21]$.

The more restricted use, the selection of appropriate antibacterial agents and the use of ribosomal immunotherapy for OME would almost certainly slow the rise in resistance.

The positive results obtained in the present study with Immucytal are in agreement with the findings of the other principal investigations of ribosomal immunotherapy and strongly support its use in paediatric patients with recurrent respiratory infections.

The results of the treatment show the therapeutic effectiveness of this approach in the prevention of otitis media in children. We can expect a long-term follow up of these children: the brevity and absence of collateral effects suggest the possibility to repeat the therapeutic trial in order to strengthen the effectiveness of the drug.

\section{References}

[1] J.A. Rosenfeld, G. Clarity, Acute otitis media in children, Prim. Care 23 (1996) 67786

[2] A.W. Cripps, J. Kyd, Bacterial otitis media: current vaccine development strategies, Immunol. Cell Biol. 81 (2003) 4651.

[3] R. Mora, M. Barbieri, G.C. Passali, A. Sovatzis, F. Mora, M.P. Cordone, A preventive measure for otitis media in children with upper respiratory tract infection, Int. J. Pediatr. Otorhinolaryngol. 63 (2002) 111-118.

[4] T. Heikkinen, T. Chonmaitree, Increasing importance of viruses in acute otitis media, Ann. Med. 32 (2000) 157163.

[5] T. Heikkinen, T. Chonmaitree, Importance of respiratory viruses in acute otitis media, Clin. Microbiol. Rev. 16 (2003) 230-241.

[6] T. Heikkinen, The role of respiratory viruses in otitis media, Vaccine 19 (2002) 51-55.

[7] A. Marchese, F. Ardito, G. Fadda, R. Fontana, G. Lo Cascio, F. Marcheti, G. Nicoletti, A.M. Speciale, A.M. Schito, Sentinel Project 2000: an update on the prevalence of antimicrobial resistance in common bacterial pathogens of respiratory tract infections circulating in Italy, GIMMOC 6 (2002) $168-185$

[8] A. Fiocchi, R. Arancio, P. Cinquepalmi, M. Sala, G.V. Zuccotti, E. Riva, M. Giovannini, Recurrent respiratory infections in childhood: experience with a bacterial extract plus bacterial ribosomes (Immucytal), J. Int. Med. Res. 18 (1990) 50-60.

[9] M. de Martino, L. Galli, A. Vierucci, May children with recurrent respiratory infections be a test bed of immunomodulators, Pharmacol. Res. 26 (1992) 156-159.

[10] G. Dietrich, M. Griot-Wenk, I.C. Metcalfe, A.B. Lang, J.F. Viret, Experience with registered mucosal vaccines, Vaccine 21 (2003) 678-683.

[11] M.C. Bene, G.C. Faure, From Peyer's patches to tonsils. Specific stimulation with ribosomal immunotherapy, Drugs 54 (1997) 24-28.

[12] K. Eriksson, J. Holmgren, Recent advances in mucosal vaccines and adjuvants, Curr. Opin. Immunol. 14 (2002) 666672

[13] J. Clot, Pharmacology of ribosomal immunotherapy, Drugs 54 (1997) 33-36.

[14] P. Allavena, E. Annalaura, A. Pirelli, L. Licciardello, A. Mantovani, Stimulation of cytotoxic and non-cytotoxic functions of natural killer cells by bacterial membrane proteoglycans and ribosomes, Int. J. Immunopharmacol. 11 (1989) 29-34.

[15] C. Roques, M.N. Frayret, J. Luc, G. Michel, R.M. Rouquet, P. Leophonte, G. Dutau, Immunostimulant effects on granulocyte functions during an acute respiratory infection, Dev. Biol. Stand. 77 (1992) 183-187.

[16] M.N. Kolopp-Sarda, M.E. Bene, J.M. Allaire, A.M. Perruchet, G.C. Faure, Kinetics of specific salivary IgA responses in man after oral challenge by ribosomal immunostimulant, Int. J. Immunopharmacol. 19 (1997) 181186.

[17] H. Takahashi, I. Honjo, A. Fujita, Endoscopic findings at the pharyngeal orifice of the eustachian tube in otitis media with effusion, Eur. Arch. Otorhinolaryngol. 253 (1996) 4244.

[18] S.A. Moodly, C.M. Alper, W.J. Doyle, Daily tympanometry in children during the cold season: association of otitis media with upper respiratory tract infections, Int. J. Pediatr. Otorhinolaryngol. 45 (1998) 143-150. 
[19] M. Rutishauser, P. Pitzke, G. Grevers, A. van Aubel, U. Elsasser, A. Kammereit, Use of a polyvalent bacterial lysate in patients with recurrent respiratory tract infections: results of a prospective, placebo-controlled, randomized, double-blind study, Adv. Ther. 15 (1998) 330341.
[20] C.E. Johnson, S. Belman, The role of antibacterial therapy of acute otitis media in promoting drug resistance, Paediatr. Drugs 3 (2001) 639-647.

[21] S.L. Block, Management of acute otitis media in the 1990s: the decade of resistant pneumococcus, Paediatr. Drugs 1 (1999) 31-50.

\section{Available online at www.sciencedirect.com science@Dinect.}

